\title{
Plasticity in the Structure of Visual Space
}

\author{
DChen Song, Andrew M. Haun, and Giulio Tononi
}

DOI:http://dx.doi.org/10.1523/ENEURO.0080-17.2017

Department of Psychiatry, University of Wisconsin-Madison, Madison, WI 53719

\section{Visual Abstract}

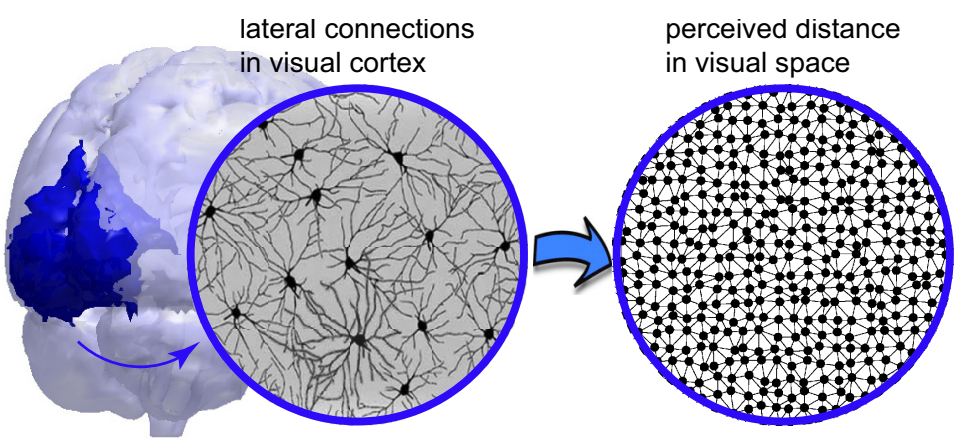

Visual space embodies all visual experiences, yet what determines the topographical structure of visual space remains unclear. Here we test a novel theoretical framework that proposes intrinsic lateral connections in the visual cortex as the mechanism underlying the structure of visual space. The framework suggests that the strength of lateral connections between neurons in the visual cortex shapes the experience of spatial relatedness between locations in the visual field. As such, an increase in lateral connection strength shall lead to an increase in perceived relatedness and a contraction in perceived distance. To test this framework through human psychophysics experiments, we used a Hebbian training protocol in which two-point stimuli were flashed in synchrony at separate locations in the visual field, to strengthen the lateral connections between two separate groups of neurons in the visual cortex. After training, participants experienced a contraction in perceived distance. Intriguingly, the perceptual contraction occurred not only between the two training locations that were linked directly by the changed connections, but also between the outward untrained locations that were linked indirectly through the changed connections. Moreover, the effect of training greatly decreased if the two training locations were too close together or too far apart and went beyond the extent of lateral connections. These findings suggest that a local change in the strength of lateral connections is sufficient to alter the topographical structure of visual space.

Key words: Lateral connections; visual plasticity; visual space

\section{Significance Statement}

Given that visual space underlies visual perception, it is easy to take its topographical structure for granted. Indeed, most studies focus on object or feature perception that happens within visual space, without first considering the structure of visual space itself. Here we studied plasticity in the structure of visual space. We found that a local strengthening of lateral connections between retinotopically tuned visual cortical neurons, induced by synchronized, repetitive presentation of two-point stimuli, could lead to a contraction in perceived distance and a change in visual space structure. We propose lateral connections in the visual cortex as the mechanism that relates locations perceptually and shapes the structure of visual space.

Received March 10, 2017; accepted June 1, 2017; First published June 05, 2017.

Authors report no conflict of interest.
Author contributions: CS conducted the experiment. CS and $\mathrm{AH}$ designed the experiment and analyzed the data. $\mathrm{CS}, \mathrm{AH}$, and $\mathrm{GT}$ conceived the study and wrote the paper. 


\section{Introduction}

More than a third of the human cerebral cortex is occupied by retinotopic maps of the visual field, in which individual neurons respond to specific locations in the visual field and nearby neurons to nearby locations (Sereno et al., 1995; Brewer and Barton, 2012; Katzner and Weigelt, 2013; Wang et al., 2014). This mapping between visual field and cortex can explain behavioral aspects of spatial localization (Rose, 1999). However, it is unclear what underlies the experience of spatial relations between locations: why do locations feel ordered in the specific way they do, apart from our abilities to behaviorally localize targets?

Whereas individual neurons in the visual cortex respond only to limited locations in the visual field, the lateral connections between these retinotopically tuned neurons instead allow distinct locations to be related. We hypothesize that the strength of lateral connections between neurons in the visual cortex determines the degree of perceived relatedness between locations in the visual field (Tononi, 2014; Tononi et al., 2016). This hypothesis explains how the organization of lateral connections, where the connection strength between neurons decays with their cortical separation (Clarke, 1994; Das and Gilbert, 1999), naturally gives rise to the topographical structure of visual space, where the perceived relatedness between locations decays with their visual field separation. Importantly, this hypothesis predicts that a change in the strength of lateral connections should alter the structure of visual space and affect the perceived relatedness between locations. Specifically, an increase in lateral connection strength should lead to an increase in perceived relatedness and a contraction in perceived distance. Moreover, the hypothesis predicts that the perceptual changes should occur not only between locations linked directly by the changed connections, but also between locations linked indirectly through the changed connections.

To test this hypothesis, we used a Hebbian training protocol in which two-point stimuli were flashed in synchrony at separate locations in the visual field, to induce a short-term increase in the strength of lateral connections between two separate groups of neurons in the visual cortex (Yao and Dan, 2001; Fu et al., 2002, 2004; Caporale and Dan, 2008). A successful induction of synaptic plasticity requires the presence of direct connections between the two neuronal groups (Ganguly et al., 2000; Li et al., 2004). It follows that, depending on the

The study was supported by Templeton Foundation (GT).

Acknowledgments: We thank Brad Postle for lab and experiment facilities and Jason Samaha, Melanie Boly, William Marshall, Chiara Cirelli, and Larissa Albantakis for valuable discussions.

Correspondence should be addressed to Giulio Tononi, University of Wisconsin-Madison, 6001 Research Park Blvd., Madison, WI 53719. E-mail: gtononi@wisc.edu.

DOI:http://dx.doi.org/10.1523/ENEURO.0080-17.2017

Copyright (C) 2017 Song et al.

This is an open-access article distributed under the terms of the Creative Commons Attribution 4.0 International license, which permits unrestricted use, distribution and reproduction in any medium provided that the original work is properly attributed. distribution of lateral connection length, there should be an optimal separation between the two training locations at which the net increase in lateral connection strength is maximal and the contraction in perceived distance is accordingly maximal. At longer separation, the two neuronal groups would not be effectively connected; at shorter separation, the two neuronal groups could be partially overlapping; either way, the number of lateral connections involved and the net change in lateral connection strength would be less (Fig. 1). We therefore varied the separation between the two training locations from run to run and measured the effect of training on the perceived distance between the training locations and the perceived distance between the outward untrained locations.

\section{Materials and Methods}

\section{Participants}

Thirty healthy young adults (18 female) gave written informed consent to participate in the experiment that was approved by the Institutional Review Board of the University of Wisconsin-Madison. The experiments were conducted in a dark room with the display (ASUS PG278Q, 27-inch, $2560 \times 1440$ pixels, $120 \mathrm{~Hz}$ ) providing the only significant source of light. Throughout the experiments, participants maintained central fixation and viewed the visual stimuli from a chin rest at a distance of $25 \mathrm{~cm}$.

\section{Procedures}

Each experiment run began with two testing sessions and was followed by three alternating cycles of training and testing sessions. In the training session, we used a Hebbian training protocol that was adapted from animal experiments and has been shown to strengthen the lateral connections in cat primary visual cortex (Yao and Dan, 2001; Fu et al., 2002, 2004; Caporale and Dan, 2008). To target lateral connections in human primary visual cortex (V1), we utilized the mirror-symmetry of human retinotopic organization. V1 is the only early visual cortical region whose ventral part (representation of upper visual field) and dorsal part (representation of lower visual field) are contiguous and are directly connected by lateral connections (Sereno et al., 1995; Brewer and Barton, 2012; Wang et al., 2014). In contrast, the ventral and dorsal parts of other early visual cortical regions (e.g., V2, V3) are segregated by $\mathrm{V} 1$ and may have a different organization of lateral connections. We therefore placed the two training locations in the upper and lower visual fields, to activate two separate groups of neurons in the ventral and dorsal parts of the visual cortex, respectively. Specifically, we flashed two synchronized, vertically separated point stimuli (5-pixel diameter) in the right hemifield at a rate of $\mathrm{ON}$ for $8.33 \mathrm{~ms}$, OFF for $425 \mathrm{~ms}$, and 275 cycles per training session (120 s). Participants were instructed to passively view the stimuli and minimize eye blinks.

In the testing session, we used a psychophysical match-to-standard protocol to measure the perceived distance between testing locations in the visual field. In each trial, two pairs of vertically separated dots (2-pixel 


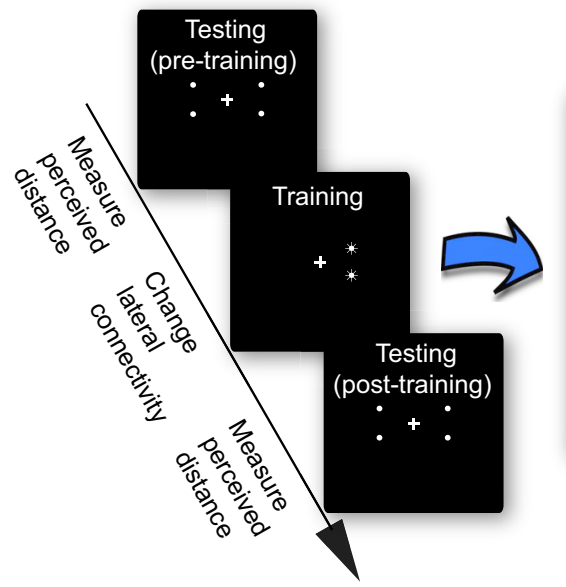

Training at shorter separation

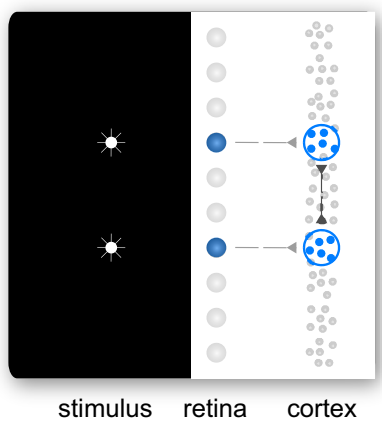

Training at optimal separation

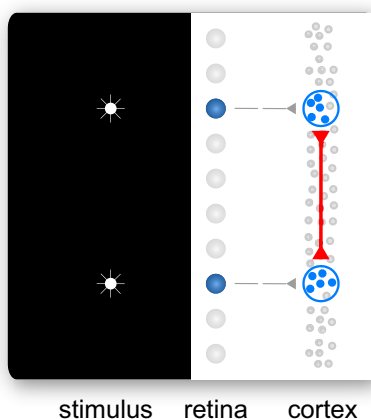

Training at longer separation

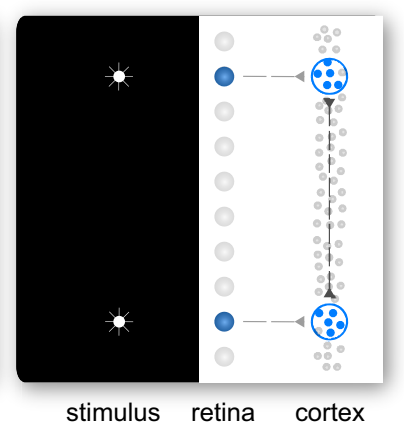

Fig. 1. Experiment design. Each experiment run contained pretraining testing, training, and posttraining testing sessions. In the training session, we used synchronized, repetitive presentation of two-point stimuli to strengthen the lateral connections between two retinotopically tuned neuronal groups. Because the successful induction of synaptic plasticity requires the presence of direct connections between the two neuronal groups, there should be an optimal separation between the two-point stimuli for changing the lateral connection strength. At longer separation, the two neuronal groups would not be effectively connected; at shorter separation, the two neuronal groups could be partially overlapping; either way, the number of lateral connections involved and the net change in lateral connection strength would be less. In the testing session, we used a match-to-standard protocol to measure the perceived distance. Participants adjusted the physical separation of a dot pair in the untrained hemifield to match the perceived distance of a dot pair in the trained hemifield. The difference between the pre- and posttraining matches was taken to quantify the change in perceived distance and the effect of training.

diameter) were presented simultaneously for $200 \mathrm{~ms}$ in the two hemifields. Participants were instructed to report which dot pair appeared more separated or whether the two dot pairs appeared equally separated. The separation between the dot pair in the right hemifield was fixed at a standard value, and that in the left hemifield was adjusted by a one-up one-down double-interleaved staircase with a step size of 0.1 degrees. A total of 108 trials were obtained in two sessions of 54 trials each for the pretraining testing and three sessions of 36 trials each for the posttraining testing. The data were fitted with a logistic psychometric function to measure the point of subjective equality (PSE) where the two dot pairs appeared equally separated. The difference between the pre- and posttraining PSEs was taken to quantify the training-induced change in perceived distance.

Each participant took part in seven experiment runs. A single experiment run lasted 10 min and was followed by a 10-min compulsory rest to facilitate the recovery of pretraining baseline and minimize the accumulation of training effect across runs. In different experiment runs, we used training locations separated by $3.2,3.6,4.0,4.4$, $4.8,5.2$, or 5.6 degrees (one run each in random order) at a horizontal eccentricity of 6 degrees. The separations between testing locations were $0,0.4$, or 0.8 degrees larger than the separations between training locations. These visual field separations correspond to a cortical separation of 10-14 mm in human V1 (Schira et al., 2007) and overlap with the reported extent of lateral connections in primate visual cortex (Ringo, 1991; Burkhalter et al., 1993; Kaas, 2000; Levitt and Lund, 2002; Voges, et al., 2010; Ahmed et al., 2012; Lyon et al., 2014). Moreover, at an eccentricity of 6 degrees, the number of neurons activated by a point stimulus (cortical point image) should be minimal (Van Essen et al., 1984; Harvey and Dumoulin, 2011), thereby favoring the activation of distinct groups of neurons. The choices of training parameters were determined from pilot experiments, where we tested the effect of stimulus eccentricity $(4,6,8$ degrees), size (5-, 15-, 30-pixel diameter), pattern (two-point, two patches of dots, two checkboard wedges), and flashing profile (in synchrony or with interstimulus interval of 8.33, $16.67,25.00,33.33,41.67,50.00,58.33,66.67,216.67$ $\mathrm{ms}$ ), through 276 experiment runs in three participants. Of the pilot parameters, those that favored focal activation of two separate groups of neurons (e.g., two-point as opposed to two patches of dots or two checkboard wedges) were used for formal experiments.

\section{Analysis}

We quantified the training induced changes in the perceived distance between testing locations, and the dependence of training effects on the separation between training locations. We first performed repeated-measures ANOVA on the raw data, with pre-/posttraining and training separation as the within-subject factors. We then estimated how the training effect and its dependence on training separation differed across participants, presumably as a consequence of interindividual differences in visual cortical architecture (Andrews et al., 1997; Dougherty et al., 2003; Schwarzkopf et al., 2011, 2012; Song et al., 2011, 2013a, 2013b, 2015). For each participant, we fitted the training effect with a Gaussian function of the training separation and took the separation closest to the Gaussian peak as the optimal training separation. This procedure allowed us to calculate the group average after aligning each participant's data to their optimal training separation. 
Training at shorter separation

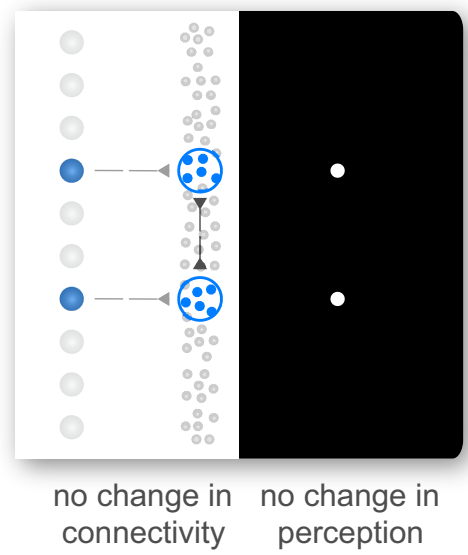

Training at optimal separation

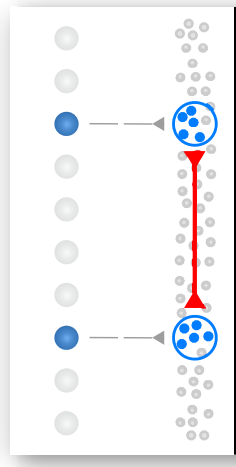

changed

connectivity

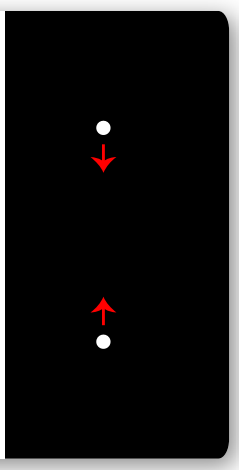

changed perception
Training at longer separation

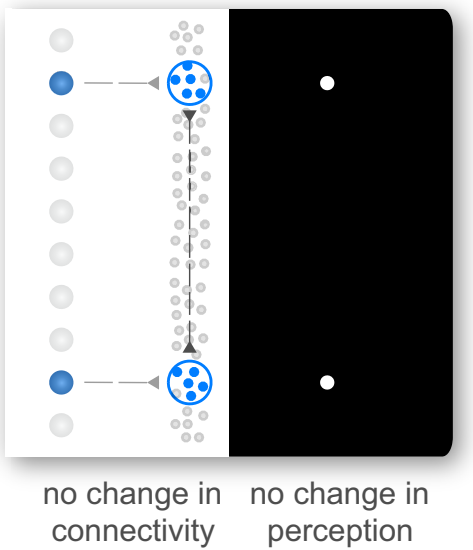

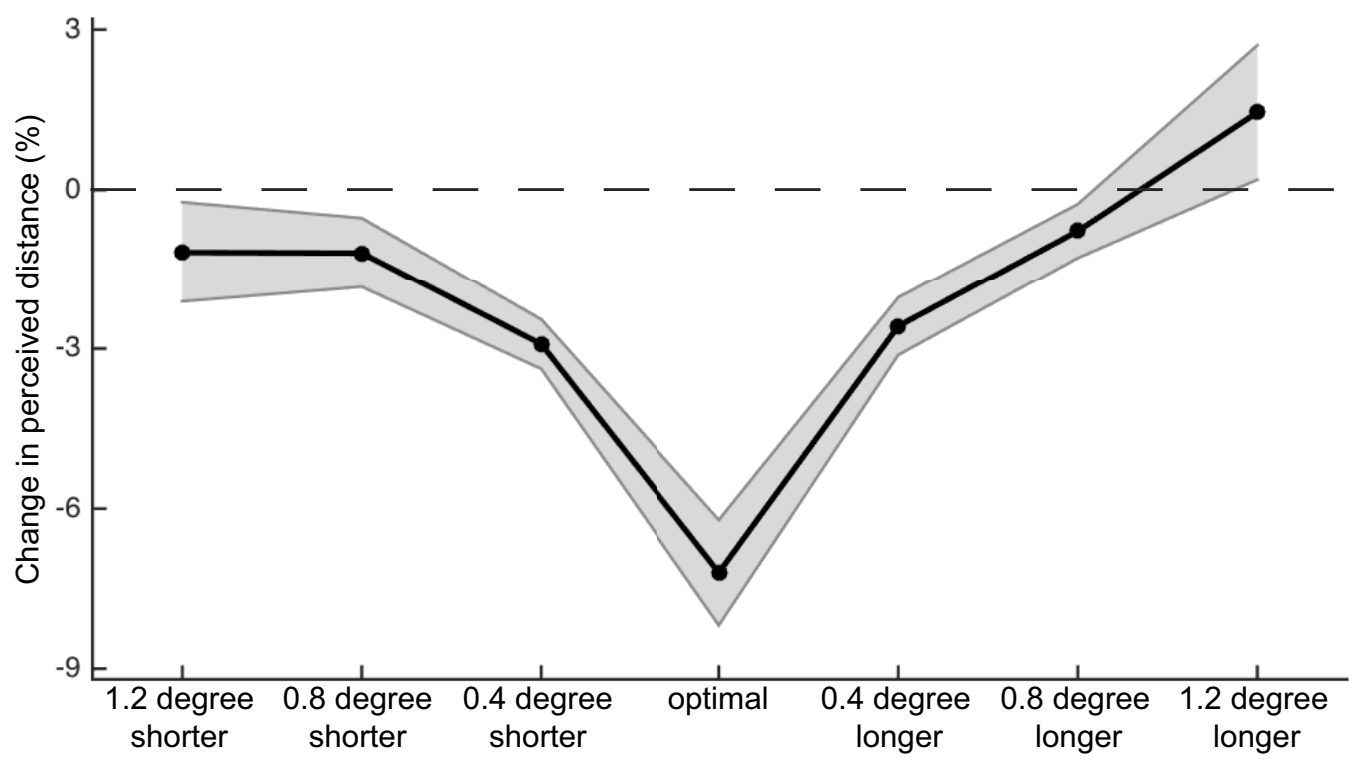

Separation between the training locations

Fig. 2. Change in perceived distance depends on training separation. The induction of synaptic plasticity and the change in lateral connection strength should be dependent on the separation between training locations. We measured the effect of training for a range of training separations. We observed a maximal contraction in perceived distance when training at a separation of 4.4 degrees. The contraction declined when training at shorter or longer separations. Black line, group average; shaded area, SEM ( $n=30$ ).

\section{Results}

After training, we observed a significant contraction in the perceived distance between the training locations, with the degree of contraction dependent on the training separation (repeated-measures ANOVA; effect of training on perceived distance: $F(1,29)=41.473$, $\mathrm{p}<$ $10^{-6}$; quadratic trend in interaction between training effect and training separation: $F(1,29)=7.632, p=$ 0.010 . The contraction was maximal at a training separation of 4.4 degrees, which corresponds to a cortical separation of $12 \mathrm{~mm}$ in human V1 (Schira et al., 2007) and falls within the extent of $\mathrm{V} 1$ lateral connections (Ringo, 1991; Burkhalter et al., 1993; Kaas, 2000; Levitt and Lund, 2002; Voges, et al., 2010; Ahmed et al., 2012; Lyon et al., 2014). Because of the mirror-symmetry of human retinotopic organization, V1 is the only early visual cortical region whose ventral part (representation of upper visual field) and dorsal part (representation of lower visual field) are contiguous, whereas the ventral and dorsal parts of other early visual cortical regions (e.g., V2, V3) are segregated by V1 (Sereno et al., 1995; Brewer and Barton, 2012; Wang et al., 2014). In these regions (e.g., V2, V3), a visual field separation of 4.4 degrees across horizontal midline would correspond to a cortical separation much larger than $12 \mathrm{~mm}$ and would fall outside the extent of lateral connections. The observation of a maximal training effect at a 4.4-degree visual field separation thus suggests the existence of an optimal separation at which the net increase in V1 lateral connection strength is maximal. 
Training at optimal separation Testing at training locations

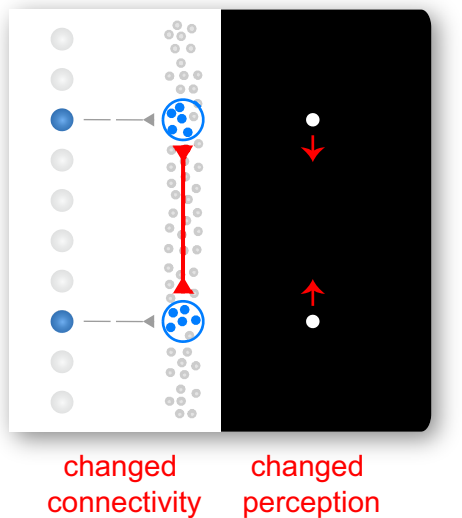

$\mathrm{T}(29)=7.3, \mathrm{p}<10^{-7}$

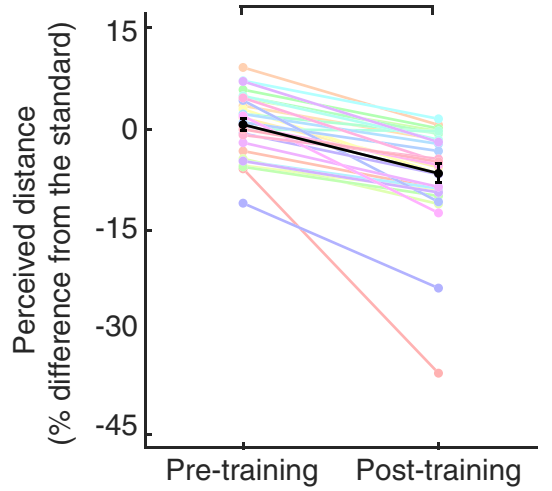

Training at optimal separation Testing at outward untrained locations
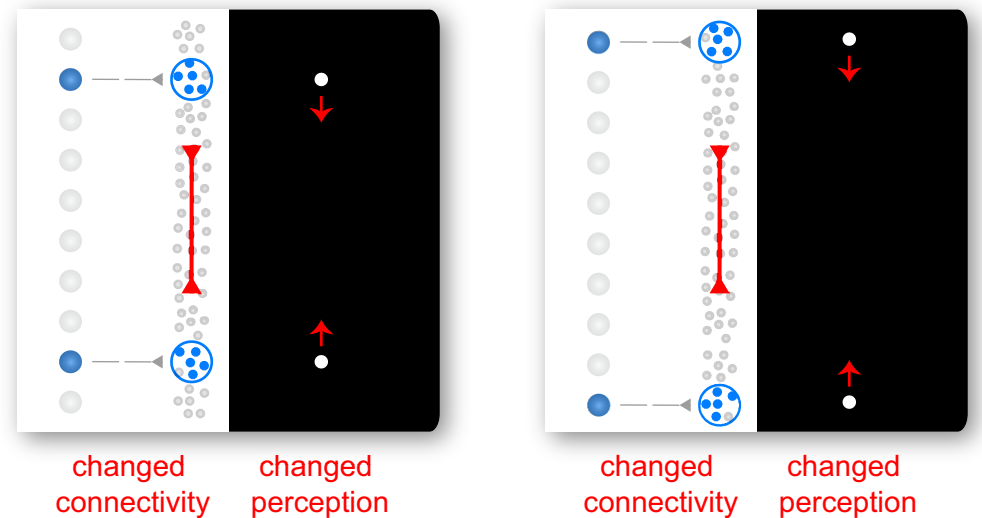
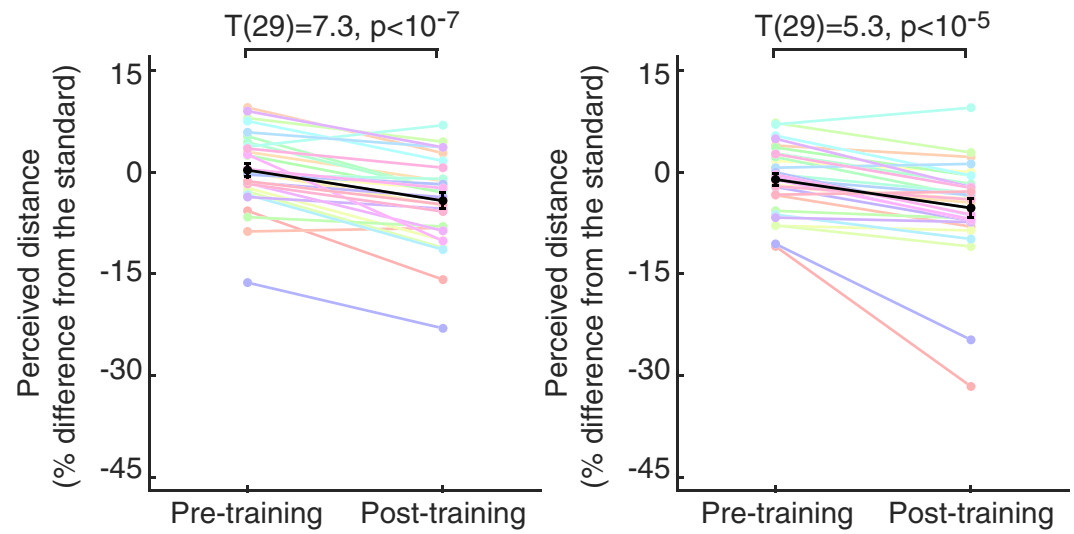

Fig. 3. Change in perceived distance between untrained locations. A change in the strength of lateral connections should affect the perceived distance, not only between the training locations, but also between the untrained locations that span the training locations. We measured the effect of training for a range of testing locations. After training at the optimal separation, the perceived distance between the testing locations at 0.4 and 0.8 degrees outward from the training locations was significantly contracted. Black line, group average with SEM $(n=30)$; colored lines, individual participants. Paired-sample $t$ tests are shown.

Because the surface area of $\mathrm{V} 1$ and the length of $\mathrm{V} 1$ lateral connections both exhibit considerable interindividual variability (Andrews et al., 1997; Dougherty et al., 2003; Schwarzkopf et al., 2011, 2012; Song et al., 2011, 2013a, 2013b, 2015), we expected the optimal training separation to vary from participant to participant. To account for the influence of this interindividual variability on the calculation of group average, we aligned each participant's data to their optimal separation. The aligned group average (Fig. 2) revealed a $7.2 \%$ contraction of perceived distance when training at the optimal separation, which decreased to $2.6 \%$ when training at \pm 0.4 degrees from the optimal separation and $0.8 \%$ when training at \pm 0.8 degrees from the optimal separation. Similar results were obtained from the prealigned raw data, which revealed a group average of $3.5 \%$ contraction at the training separation of 4.4 degrees and a significant decrease to $1.1 \%$ when training at \pm 1.2 degrees away (as reflected by the significant quadratic trend in the ANOVA).

The hypothesis that lateral connections underlie the structure of visual space further suggests that the perceived distance should be changed not only between training locations, but also between outward untrained locations that are linked indirectly by the changed connections (Fig. 3). In line with this prediction, after training at the optimal separation, the perceived distance between the testing locations at 0.4 and 0.8 degrees outward from the training locations was contracted by a significant amount of $4.2 \%\left(t(29)=7.3, p<10^{-7}\right)$ and $3.6 \%(t(29)=$ $\left.5.3, p<10^{-5}\right)$, respectively. Moreover, the contraction $(4.2 \%, 3.6 \%)$ produced by training at the optimal separation was even larger than the contraction $(2.6 \%, 0.8 \%)$ produced by training at these testing locations, illustrating again that the effect of training is very weak at nonoptimal training separation.

\section{Discussion}

Taken together, these findings suggest that a local strengthening of lateral connections induced by synchronized, repetitive presentation of two-point stimuli can lead to a contraction in perceived distance. More broadly, they suggest that lateral connections may underlie the topographical structure of visual space. The training protocol used in our study was adapted from animal studies and 
has been shown to strengthen lateral connection between neurons in cat V1 (Yao and Dan, 2001; Fu et al., 2002, 2004; Caporale and Dan, 2008). Because of the fine spatial scale over which the changes are expected to happen $(\sim 10 \mathrm{~mm})$ and the coarse spatial resolution of neuroimaging measures, it is difficult to ascertain the exact neural-level changes induced by the protocol in human participants. The protocol may have affected feedforward thalamocortical connections and changed receptive fields or response gains at the training locations (Kohler and Wallach, 1944; DeAngelis et al., 1995; Ganguly et al., 2000; Eyding et al., 2002; Hisakata et al., 2016). Similarly, we cannot rule out the training-induced changes in attention and feedback connections from fronto-parietal cortices to visual cortex (Anton-Erxleben et al., 2007; Klein et al., 2016).

Such feedforward or feedback mechanisms have been proposed to account for the perceptual changes induced by repetitive exposure to a visual stimulus and the transfer of perceptual effects across locations, features, or tasks (Fahle et al., 1995; Dill and Fahle, 1997; Goldstone, 1998; Sasaki et al., 2010; Zhang and Li, 2010). Usually, the transfer of perceptual effects to an untrained location is taken to indicate a feedback mechanism, whereas the dependence of perceptual effects on a retinotopic frame is taken to support a feedforward mechanism. However, neither feedforward nor feedback mechanisms can account for a U-shaped relation between the degree of perceptual changes and the separation of training locations, as was found here (Fig. 2). Moreover, a feedforward mechanism cannot explain the contraction in perceived distance between the untrained locations, as we also found (Fig. 3). Instead, our findings are exactly as predicted by the hypothesis that lateral connections linking neighboring neurons in the visual cortex shape the structure of experienced space (Fig. 1).

Given that visual space underlies visual perception, it is easy to take its topographical structure for granted. Indeed, most studies of visual perception focus on object or feature perception that happens within visual space, without considering the structure of space itself. One exception is a recent report of a paradoxical co-occurrence between decreased perceived density of dot textures and contracted perceived distance between dot pairs after adaptation to a large-field random dot stimulus (Hisakata et al., 2016). This finding cannot be explained by changes in neuronal response properties, and the authors instead proposed the scaling of an "internal metric" in the visual cortical system that relates distinct locations and specifies perceived distance (Hisakata et al., 2016). Our study suggests that this internal metric is provided by the organization and strength of lateral connections in retinotopic visual cortex.

Although the lateral connections are present throughout the visual cortex (Kaas, 2000; Levitt and Lund, 2002), different visual cortical regions may play different roles in the structure of visual space. Because of the cortical convergence, regions higher up in the visual hierarchy usually occupy less cortical area and have fewer neurons (Haug, 1987; Dougherty et al., 2003). Moreover, individual neurons in these visual cortical regions inherit the aggregate receptive fields of their multiple feedforward inputs (Sereno et al., 1995; Brewer and Barton, 2012; Katzner and Weigelt, 2013; Wang et al., 2014). By contrast, V1 at the bottom of the visual hierarchy has the largest cortical surface area, the largest number of neurons, and the smallest receptive fields (Andrews et al., 1997; Dougherty et al., 2003; Schwarzkopf et al., 2011, 2012; Song et al., 2011, 2013a, 2013b, 2015). The lateral connections in higher-up visual cortical regions will therefore span larger separations in the visual field and specify a coarser visual space, whereas the lateral connections in V1 will specify a finer visual space. These different spatial scales may jointly ensure a robust structure of visual space. Following on this proposal, an important next step would be to apply protocols that can target different visual cortical regions and selectively strengthen or weaken connections, such as theta burst transcranial magnetic stimulation (Huang et al., 2005; Salminen-Vaparanta et al., 2012; Rahnev et al., 2013; Silson et al., 2013), to examine the roles of individual visual cortical regions in the structure of visual space.

\section{References}

Ahmed B, Cordery PM, McLelland D, Bair W, Krug K (2012) Longrange clustered connections within extrastriate visual area V5/MT of the rhesus macaque. Cereb Cortex 22:60-73. CrossRef

Andrews TJ, Halpern SD, Purves D (1997) Correlated size variations in human visual cortex, lateral geniculate nucleus, and optic tract. J Neurosci 17:2859-2868.

Anton-Erxleben K, Henrich C, Treue S (2007) Attention changes perceived size of moving visual patterns. J Vision 7:1-9. CrossRef

Brewer AA, Barton B (2012). Visual Field Map Organization in Human Visual Cortex. Visual Cortex: Current Status and Perspectives, S. Molotchnikoff (ed.), InTech, doi:10.5772/51914.

Burkhalter A, Bernardo KL, Charles V (1993) Development of local circuits in human visual cortex. J Neurosci 13:1916-1931. Medline

Caporale N, Dan Y (2008) Spike timing-dependent plasticity: a Hebbian learning rule. Annu Rev Neurosci 31:25-46. CrossRef

Clarke S (1994) Association and intrinsic connections of human extrastriate visual cortex. Proc R Soc Lond B Biol Sci 257:87-92. CrossRef

Das A, Gilbert CD (1999) Topography of contextual modulations mediated by short-range interactions in primary visual cortex. Nature 399:655-661. CrossRef Medline

DeAngelis GC, Anzai A, Ohzawa I, Freeman RD (1995) Receptive field structure in the visual cortex: does selective stimulation induce plasticity? PNAS 92:9682-9686. CrossRef

Dill M, Fahle M (1997) The role of visual field position in pattern discrimination learning. Proc R Soc Lond B Biol Sci 264:10311036. CrossRef

Dougherty RF, Koch VM, Brewer AA, Fischer B, Modersitzki J, Wandell BA (2003) Visual field representations and locations of area $\mathrm{V} 1 / 2 / 3$ in human visual cortex. J Vis 2003:586-598.

Eyding D, Schweigart G, Eysel UT (2002) Spatio-temporal plasticity of cortical receptive fields in response to repetitive visual stimulation in the adult cat. Neuroscience 112:195-215. CrossRef

Fahle M, Edelman S, Poggio T (1995) Fast perceptual learning in hyperacuity. Vis Res 35:3003-3013. Medline

Fu YX, Djupsund K, Gao H, Hayden B, Shen K, Dan Y (2002) Temporal specificity in the cortical plasticity of visual space representation. Science 296:1999-2003. CrossRef

Fu YX, Shen Y, Gao H, Dan Y (2004) Asymmetry in visual cortical circuits underlying motion-induced perceptual mislocalization. J Neurosci 24:2165-2171. CrossRef 
Ganguly K, Kiss L, Poo M (2000) Enhancement of presynaptic neuronal excitability by correlated presynaptic and postsynaptic spiking. Nat Neurosci 3:1018-1026. CrossRef

Goldstone RL (1998) Perceptual learning. Annu Rev Psychol 49:585612. CrossRef Medline

Harvey BM, Dumoulin SO (2011) The relationship between cortical magnification factor and population receptive field size in human visual cortex: constancies in cortical architecture. J Neurosci 31: 13604-13612. CrossRef

Haug $H$ (1987) Brain sizes, surfaces, and neural sizes of the cerebral cortex: a stereological investigation of man and his variability and a comparison with some mammals. Am J Anat 180:126-142. CrossRef

Hisakata R, Nishida S, Johnston A (2016) An adaptable metric shapes perceptual space. Curr Biol 26:1911-1915. CrossRef Medline

Huang YZ, Edwards MJ, Rounis E, Bhatia KP, Rothwell JC (2005) Theta burst stimulation of the human motor cortex. Neuron 45: 201-206. CrossRef

Kaas JH (2000) Why is brain size so important: design problems and solutions as neocortex gets bigger or smaller. Brain Mind 1:7-23. CrossRef

Katzner S, Weigelt S (2013) Visual cortical networks of mice and men. Curr Opin Neurobiol 2013:202-206. CrossRef

Klein BP, Paffen CL, Te Pas SF, Dumoulin SO (2016) Predicting bias in perceived position using attention field models. J Vis 16:1-15. CrossRef Medline

Kohler W, Wallach HH (1944) Figural aftereffects: an investigation of visual processes. Proc Am Philos Soc 88:269-357.

Levitt JB, Lund JS (2002). Intrinsic Connections in Mammalian Cerebral Cortex. Boca Raton, FL: CRC Press.

Li C, Lu J, Wu C, Duan S, Poo M (2004) Bidirectional modification of presynaptic neuronal excitability accompanying spike timingdependent synaptic plasticity. Neuron 41:257-268. CrossRef

Lyon D, Liu YJ, Arreola M, Coleman C (2014) Very-long-range disynaptic $\mathrm{V} 1$ connections through layer 6 pyramidal neurons revealed by transneuronal tracing with rabies virus. Eye Brain 45:45-56.

Rahnev D, Kok P, Munneke M, Bahdo L, de Lange FP, Lau H (2013) Continuous theta burst transcranial magnetic stimulation reduces resting state connectivity between visual areas. J Neurophysiol 110:1811-1821. CrossRef

Ringo JL (1991) Neuronal interconnection as a function of brain size. Brain, Behav Evol 38:1-6. Medline

Rose D (1999) The historical roots of the theories of local signs and labelled lines. Perception 28:675-685. CrossRef Medline

Salminen-Vaparanta N, Noreika V, Revonsuo A, Koivisto M, Vanni S (2012) Is selective primary visual cortex stimulation achievable with TMS? Hum Brain Mapp 33:652-665. CrossRef

Sasaki Y, Nanez JE, Watanabe T (2010) Advances in visual perceptual learning and plasticity. Nat Rev Neurosci 11:53-60. CrossRef Medline
Schira MM, Wade AR, Tyler CW (2007) Two-dimensional mapping of the central and parafoveal visual field to human visual cortex. $J$ Neurophysiol 97:4284-4295. CrossRef Medline

Schwarzkopf DS, Song C, Rees G (2011) The surface area of human V1 predicts the subjective experience of object size. Nat Neurosci 14:28-30. CrossRef Medline

Schwarzkopf DS, Robertson DJ, Song C, Barnes GR, Rees G (2012) The frequency of visually induced gamma-band oscillations depends on the size of early human visual cortex. J Neurosci 32: 1507-1512. CrossRef

Sereno MI, Dale AM, Reppas JB, Kwong KK, Belliveau JW, Brady TJ, Rosen BR, Tootell RB (1995) Borders of multiple visual areas in humans revealed by functional magnetic resonance imaging. Science 268:889-893. CrossRef

Silson EH, McKeefry DJ, Rodgers J, Gouws AD, Hymers M, Morland AB (2013) Specialized and independent processing of orientation and shape in visual field maps LO1 and LO2. Nat Neurosci 16: 267-269. CrossRef

Song C, Schwarzkopf DS, Kanai R, Rees G (2011) Reciprocal anatomical relationship between primary sensory and prefrontal cortices in the human brain. J Neurosci 31:9472-9480. CrossRef

Song C, Schwarzkopf DS, Lutti A, Li B, Kanai R, Rees G (2013a) Effective connectivity within human primary visual cortex predicts interindividual diversity in illusory perception. J Neurosci 33: 18781-18791. CrossRef

Song C, Schwarzkopf DS, Rees G (2013b) Variability in visual cortex size reflects tradeoff between local orientation sensitivity and global orientation modulation. Nat Commun 4:2201. CrossRef

Song C, Schwarzkopf DS, Kanai R, Rees G (2015) Neural population tuning links visual cortical anatomy to human visual perception. Neuron 85:641-656. CrossRef

Tononi G (2014). The conscious grid. http://integratedinformationtheory. org/download/conscious_grid.pdf.

Tononi G, Boly M, Massimini M, Koch C (2016) Integrated information theory: from consciousness to its physical substrate. Nat Rev Neurosci 17:450-461. CrossRef

Van Essen DC, Newsome WT, Maunsell JH (1984) The visual field representation in striate cortex of the macaque monkey: asymmetries, anisotropies, and individual variability. Vis Rese 24:429-448. CrossRef

Voges N, Schüz A, Aertsen A, Rotter S (2010) A modeler's view on the spatial structure of intrinsic horizontal connectivity in the neocortex. Prog Neurobiol 92:277-292. CrossRef

Wang L, Mruczek RE, Arcaro MJ, Kastner S (2014) Probabilistic maps of visual topography in human cortex. Cereb Cortex 25: 3911-3931. CrossRef Medline

Yao H, Dan Y (2001) Stimulus timing-dependent plasticity in cortical processing of orientation. Neuron 32:315-323. Medline

Zhang E, Li W (2010) Perceptual learning beyond retinotopic reference frame. PNAS 107:15969-15974. CrossRef Medline 